\title{
Quality of Harvested Seed Associated with Soybean Cultivars and Herbicides Under Weed-Free Conditions
}

\author{
C. A. Bradley, Department of Plant Pathology, North Dakota State University, Fargo 58105; G. L. Hartman and L. \\ M. Wax, USDA/ARS and Department of Crop Sciences, University of Illinois; and W. L. Pedersen, Department of \\ Crop Sciences, University of Illinois, National Soybean Research Laboratory, 1101 W. Peabody Dr., Urbana 61801
}

\begin{abstract}
Bradley, C. A., Hartman, G. L., Wax, L. M., and Pedersen, W. L. 2002. Quality of harvested seed associated with soybean cultivars and herbicides under weed-free conditions. Plant Dis. 86:1036-1042.

Different herbicides were applied to soybean plants in field plots planted to different soybean cultivars located at four locations in Illinois between 1997 and 2000. Treatments varied from hand weeded to preemergence herbicides to postemergence herbicides. Soybean seeds were harvested and evaluated for different seed quality parameters. The percentage of seeds infected with Phomopsis spp. ranged from 1 to $40 \%$, and the percentage of seeds infected with Cercospora kikuchii was low, ranging from 0 to $4 \%$. Herbicides had little or no effect on seed quality parameters such as percent germination and incidence of seed pathogens or on protein and oil concentrations. Soybean seed quality was affected by Phomopsis spp. in that there were significant $(P \leq 0.05)$ inverse correlations between Phomopsis spp. incidence and percentage seed germination. It appears that Phomopsis spp. may be a more prevalent seed pathogen than $C$. kikuchii for soybean fields in central to northern Illinois.
\end{abstract}

Additional keywords: Glycine max, pod and stem blight, purple seed stain

Phomopsis seed decay of soybean (Glycine $\max (\mathrm{L}$.) Merr.), caused primarily by Phomopsis longicolla Hobbs, but also caused by other Phomopsis and Diaporthe spp., is endemic to Illinois (33). Infected seeds may have reduced germination, split more readily, and suffer other quality losses compared to noninfected seeds $(16,24,35)$. Purple seed stain of soybean, caused by Cercospora kikuchii (Matsu. \& Tomoyasu) Gardner, is a seed disease of soybean that may cause purple speckling of seed, reduced germination, and other quality losses $(27,44,46)$. These diseases also cause an economic loss to growers, in that grain buyers may discount the price paid for soybeans if seed is discolored due to infection $(34,36)$.

Herbicides have been shown to affect different seed quality factors and the presence of seed pathogens. Bowman et al. (5) found that seeds from soybean plants treated with a mixture of bentazon and

Corresponding author: W. L. Pedersen E-mail: wpederse@uiuc.edu

Mention of a trademark or proprietary product does not constitute a guarantee or warranty of the product by the U.S. Department of Agriculture and does not imply its approval to the exclusion of other products that may also be suitable.

Accepted for publication 9 May 2002.

Publication no. D-2002-0722-05R

(C) 2002 The American Phytopathological Society sethoxydim were heavier than seeds from plants growing in soil treated with a mixture of alachlor and metribuzin. Other researchers found that herbicides could cause small alterations of soybean seed components, but considered the alterations unimportant $(14,28,38)$. Weed control can affect development of seedborne diseases by indirectly influencing the microenvironment and population of alternate hosts. Less seed infection by Fusarium semitectum, C. kikuchii, Phomopsis spp., and Rhizoctonia solani have occurred in plots where weeds were controlled compared to weedy control plots $(5,8,10,12)$. Direct effects of herbicides on root and foliar soybean diseases have been examined $(3,4,6,7,9,11,31,32)$, but direct effects of herbicides on seed diseases have not been examined in weed-free conditions.

The objectives of this research were to determine the effects of herbicides and seedborne pathogens on different soybean seed quality parameters, and to determine the effect of herbicides on the presence of seedborne pathogens in soybean seeds grown in weed-free plots located throughout Illinois.

\section{MATERIALS AND METHODS}

Plot maintenance, design, and treatments. Plots were established at Champaign (1998 to 1999), DeKalb (1997 to 1998), Monmouth (1999 to 2000), and Urbana (1998 to 1999), Illinois. Soybean cultivars planted at Champaign and Urbana were Asgrow 3704 (maturity group, MG,
III), Asgrow 3904 (MG III), Jack (MG II), Pioneer 9363 (MG III), and Savoy (MG II). Cultivars planted at DeKalb were BSR 101 (MG I), Jack, Pioneer 9254 (MG II), and Savoy. Cultivars planted at Monmouth were Asgrow 3002 (MG III), Pioneer 93B01 (MG III), Pioneer 9363, and Siebens 2701 (MG II). Cultivars were chosen based on MG and regional popularity for each location. Plots were four to eight rows wide on $0.76-\mathrm{m}$ centers and 4.6 to $6.1 \mathrm{~m}$ long, and planted 19 May 1998 and 20 May 1999 at Champaign, 24 May 1999 and 15 May 2000 at Monmouth, 17 May 1997 and 27 May 1998 at DeKalb, and 17 May 1998 and 20 May 1999 at Urbana. The plots were arranged in a randomized complete block design with four replicates.

Herbicide treatments at Champaign and Urbana were a hand-weeded control, pendimethalin (Prowl, BASF Corp., Research Triangle Park, NC), a mixture of dimethenamid (Frontier, BASF Corp.) and metribuzin (Sencor, Bayer Corp., Kansas City, MO), acifluorfen (Blazer, BASF Corp.), and imazethapyr (Pursuit, BASF Corp.). Herbicide treatments at DeKalb were a hand-weeded control, pendimethalin, acifluorfen, imazethapyr, and a prepackaged mixture of acifluorfen and bentazon formulated as Galaxy (BASF Corp.). At Monmouth, all cultivars were glyphosate tolerant (Roundup Ready, Monsanto Company, St. Louis, MO), and the entire study received a treatment of glyphosate (Roundup Ultra, Monsanto Company) for weed control. The herbicide treatments at Monmouth were a glyphosate-only control, pendimethalin, acifluorfen, and imazethapyr. Pendimethalin and the mixture of dimethenamid and metribuzin were applied preemergence (PRE) immediately after planting. Glyphosate was applied postemergence (POST) when soybean plants were at the V3 growth stage (13). Acifluorfen, the acifluorfen plus bentazon mixture, and imazethapyr were applied POST when plants were at the V4 growth stage. Herbicides were applied at the normal field use rates and were: $1.39 \mathrm{~kg}$ a.i./ha pendimethalin; 1.47 and $0.42 \mathrm{~kg}$ a.i./ha dimethenamid and metribuzin, respectively; $0.42 \mathrm{~kg}$ a.i./ha acifluorfen; $0.07 \mathrm{~kg}$ a.i./ha imazethapyr; 0.19 and $0.84 \mathrm{~kg}$ a.i./ha, acifluorfen and bentazon, respectively; and $1.12 \mathrm{~kg}$ a.i./ha glyphosate. Crop oil concentrate (COC) was applied as an adjuvant 
with acifluorfen, imazethapyr, and acifluorfen + bentazon at $1 \% \mathrm{vol} / \mathrm{vol}$. Herbicides were applied using a $\mathrm{CO}_{2}$-pressurized backpack sprayer with a 3-m hand-held boom that had five 8003 flat-fan nozzles (Spraying Systems Co., Wheaton, IL) calibrated to deliver a total of 187 liters per ha at Champaign, DeKalb, and Urbana. At Monmouth, herbicides were applied with a tractor mounted sprayer with a 3-m boom that had five 8003 flat-fan nozzles calibrated to deliver a total of 140 liters per ha. Weeds that emerged after herbicides were applied were removed by hand in all plots.

Seed quality. Samples of soybean seeds from each plot were collected at harvest (13 to $14 \%$ moisture), dried under forced air at $32^{\circ} \mathrm{C}$ for 7 days so that seeds were of uniform moisture and taken to the laboratory to determine percent germination and incidence of seed pathogens. Split seeds were removed by hand, and 100 seeds from each sample were weighed to determine 100 -seed weight and placed on moistened wadded cellulose paper (Kimpak) in a seed germinator (Stults Scientific Engr. Corp., Springfield, IL) for 7 days at $90 \%$ relative humidity at $25^{\circ} \mathrm{C}$. The number of seeds germinated was then recorded. A seed was counted as germinated if the radicle was 2.5 times the length of the cotyledons. Forty seeds from each sample were surface-sterilized in a $0.5 \%$ solution of $\mathrm{NaOCl}$ for $4 \mathrm{~min}$ and then rinsed twice (4 min per rinse) in sterile distilled water. All 40 seeds were plated on potato dextrose agar (PDA, Difco Laboratories, Detroit, MI) with 10 seeds per $9-\mathrm{cm}$ petri dish. Plated seeds were then placed in an incubator for 5 days at $25^{\circ} \mathrm{C}$, and the incidence of seeds with Phomopsis spp. and C. kikuchii growing from them was recorded based on morphological characters $(2,17)$. Phomopsis spp. were typically recognized by characteristic floccose, dense, white colonies. Cercospora kikuchii was typically recognized by colonies that produced an intense maroonto-purple pigment usually growing from a purple stain on the seed coat. Seed samples (500 g) collected at Champaign and Urbana were sent to Iowa State University Grain Quality Laboratory (Ames, IA) for analysis of seed protein and oil concentrations using an Infratec 1220 series nearinfrared analyzer (Foss North America, Eden Prairie, MN).

Data analysis. Data from 1998 and 1999 collected at Champaign and Urbana were analyzed together because treatments were the same at both locations. At the other locations, years were analyzed together within each location. Years and locations were analyzed separately if there were significant $(P \leq 0.05)$ year or location by treatment interactions. Analysis of variance was conducted using the general linear models procedure (PROC GLM) of SAS (SAS Institute, Inc., Cary, NC). Location, years, and blocks were considered random effects. Fisher's least significant difference (LSD) was used at the 5\% probability level to compare means. Pearson correlation coefficients were determined using SAS (PROC CORR) for 100-seed weight, percent germination, Phomopsis spp. infected seed incidence, C. kikuchii infected seed incidence, protein concentration, and oil concentration.

\section{RESULTS}

Years and locations are presented separately due to significant $(P \leq 0.05)$ year and location by treatment interactions. Only main effects are presented, due to no significant cultivar by herbicide interactions.

Champaign. In 1998, there was significant $(P \leq 0.05)$ variation among cultivars for seed germination, 100-seed weight, incidence of Phomopsis spp., and protein and oil concentration (Table 1). Asgrow 3704 had significantly greater seed germination $(92 \%)$ than the other cultivars. Savoy had significantly greater 100 -seed weight (16 g) than the other cultivars, but also had a significantly greater incidence of Phomopsis spp. (16\%). Asgrow 3704 and 3904 and Pioneer 9363 had significantly greater protein concentration than the other cultivars (38\%). Jack had the greatest oil concentration (20\%). There were no significant differences among cultivars for $C$. kikuchii incidence. Herbicides did not have a significant effect on the seed quality parameters measured.

Table 1. Quality parameters of soybean seed harvested at Champaign, IL

\begin{tabular}{|c|c|c|c|c|c|c|c|c|}
\hline Year & Cultivar & Herbicide & $\begin{array}{c}\text { Germination } \\
(\%)^{\mathrm{a}}\end{array}$ & $\begin{array}{l}\text { Seed weight } \\
(\mathrm{g})^{\mathbf{b}}\end{array}$ & $\begin{array}{l}\text { Phomopsis } \\
\text { spp. }(\%)^{\mathrm{c}}\end{array}$ & 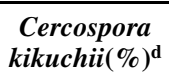 & $\begin{array}{c}\text { Protein } \\
(\%)^{\mathrm{e}}\end{array}$ & Oil (\%) \\
\hline \multirow{12}{*}{1998} & Asgrow 3704 & $\ldots$ & 92 & 13 & 3 & 0 & 38 & 19 \\
\hline & Asgrow 3904 & $\ldots$ & 87 & 15 & 5 & 1 & 38 & 19 \\
\hline & Jack & $\ldots$ & 81 & 13 & 8 & 2 & 35 & 20 \\
\hline & Pioneer 9363 & $\ldots$ & 88 & 13 & 4 & 0 & 37 & 19 \\
\hline & Savoy & $\ldots$ & 84 & 16 & 16 & 1 & 37 & 19 \\
\hline & $\operatorname{LSD}_{0.05}{ }^{\mathrm{f}}$ & $\ldots$ & 3 & 1 & 4 & $\mathrm{NS}^{\mathrm{g}}$ & 1 & 1 \\
\hline & $\ldots$ & Hand weed & 86 & 14 & 8 & 1 & 37 & 19 \\
\hline & $\ldots$ & Dimethenamid + metribuzin & 86 & 14 & 8 & 1 & 37 & 19 \\
\hline & $\ldots$ & Pendimethalin & 87 & 14 & 8 & 1 & 37 & 19 \\
\hline & $\ldots$ & Acifluorfen & 86 & 14 & 4 & 1 & 37 & 19 \\
\hline & $\ldots$ & Imazethapyr & 86 & 14 & 8 & 1 & 37 & 19 \\
\hline & $\ldots$ & $\mathrm{LSD}_{0.05}$ & NS & NS & NS & NS & NS & NS \\
\hline \multirow[t]{12}{*}{1999} & Asgrow 3704 & $\ldots$ & 94 & 15 & 4 & 0 & 37 & 18 \\
\hline & Asgrow 3904 & $\ldots$ & 90 & 15 & 6 & 2 & 38 & 17 \\
\hline & Jack & $\ldots$ & 82 & 14 & 23 & 2 & 37 & 18 \\
\hline & Pioneer 9363 & $\ldots$ & 92 & 15 & 11 & 3 & 38 & 18 \\
\hline & Savoy & $\ldots$ & 88 & 16 & 24 & 0 & 38 & 18 \\
\hline & $\operatorname{LSD}_{0.05}{ }^{\mathrm{f}}$ & $\ldots$ & 3 & NS & 5 & 1 & 1 & 1 \\
\hline & $\ldots$ & Hand weed & 88 & 14 & 14 & 2 & 38 & 18 \\
\hline & $\ldots$ & Dimethenamid + metribuzin & 88 & 15 & 12 & 1 & 38 & 18 \\
\hline & $\ldots$ & Pendimethalin & 90 & 15 & 13 & 0 & 38 & 18 \\
\hline & $\ldots$ & Acifluorfen & 90 & 16 & 14 & 2 & 38 & 18 \\
\hline & $\ldots$ & Imazethapyr & 89 & 15 & 15 & 2 & 38 & 18 \\
\hline & $\ldots$ & $\mathrm{LSD}_{0.05}$ & NS & 1 & NS & NS & NS & NS \\
\hline
\end{tabular}

\footnotetext{
a Percent seed germination.

b 100 -seed weight.

c Incidence of seed infected with Phomopsis spp.

${ }^{\mathrm{d}}$ Incidence of seed infected with Cercospora kikuchii.

e Protein concentration.

f Fisher's protected least significant difference at $\alpha=0.05$.

g Not significant at $P \leq 0.05$.
} 
In 1999 , there was significant $(P \leq 0.05)$ variation among cultivars for seed germination, incidence of Phomopsis spp., incidence of C. kikuchii, and protein and oil concentration (Table 1). Asgrow 3704 had significantly greater seed germination (94\%) than all of the other cultivars except for Pioneer 9363 (92\%). Incidence of
Phomopsis spp. was significantly greater in Savoy (24\%) and Jack (23\%) than the other cultivars. There were significant, but minute differences among cultivars for $C$.

Table 2. Quality parameters of soybean seed harvested at Urbana, IL

\begin{tabular}{|c|c|c|c|c|c|c|c|c|}
\hline Year & Cultivar & Herbicide & $\begin{array}{c}\text { Germination } \\
(\%)^{\mathrm{a}}\end{array}$ & $\begin{array}{l}\text { Seed weight } \\
(\mathrm{g})^{\mathbf{b}}\end{array}$ & $\begin{array}{l}\text { Phomopsis } \\
\text { spp. }(\%)^{\mathrm{c}}\end{array}$ & $\begin{array}{c}\text { Cercospora } \\
\text { kikuchii }(\%)^{\mathrm{d}}\end{array}$ & $\begin{array}{c}\text { Protein } \\
(\%)^{\mathrm{e}}\end{array}$ & Oil $(\%)$ \\
\hline \multirow[t]{12}{*}{1998} & Asgrow 3704 & $\ldots$ & 94 & 14 & 7 & 0 & 35 & 20 \\
\hline & Asgrow 3904 & $\ldots$ & 87 & 15 & 10 & 0 & 36 & 19 \\
\hline & Jack & $\ldots$ & 80 & 13 & 12 & 0 & 34 & 20 \\
\hline & Pioneer 9363 & $\ldots$ & 94 & 13 & 4 & 0 & 36 & 19 \\
\hline & Savoy & $\ldots$ & 88 & 18 & 15 & 0 & 36 & 19 \\
\hline & $\operatorname{LSD}_{0.05}{ }^{\mathrm{f}}$ & $\cdots$ & 3 & 1 & 5 & $\mathrm{NS}^{\mathrm{g}}$ & 1 & 1 \\
\hline & $\ldots$ & Hand weed & 89 & 14 & 10 & 0 & 36 & 19 \\
\hline & $\ldots$ & Dimethenamid + metribuzin & 88 & 14 & 9 & 0 & 36 & 19 \\
\hline & $\ldots$ & Pendimethalin & 89 & 14 & 12 & 0 & 36 & 19 \\
\hline & $\ldots$ & Acifluorfen & 88 & 15 & 10 & 0 & 36 & 20 \\
\hline & $\ldots$ & Imazethapyr & 88 & 15 & 8 & 0 & 36 & 20 \\
\hline & $\ldots$ & $\mathrm{LSD}_{0.05}$ & NS & NS & NS & NS & NS & NS \\
\hline \multirow[t]{12}{*}{1999} & Asgrow 3704 & $\ldots$ & 93 & 15 & 4 & 0 & 35 & 19 \\
\hline & Asgrow 3904 & $\ldots$ & 87 & 15 & 8 & 1 & 36 & 17 \\
\hline & Jack & $\ldots$ & 82 & 14 & 17 & 4 & 35 & 18 \\
\hline & Pioneer 9363 & $\ldots$ & 88 & 14 & 7 & 2 & 36 & 19 \\
\hline & Savoy & $\ldots$ & 83 & 17 & 16 & 0 & 37 & 19 \\
\hline & $\operatorname{LSD}_{0.05}{ }^{\mathrm{f}}$ & $\ldots$ & 3 & 1 & 6 & 2 & 1 & 1 \\
\hline & $\ldots$ & Hand weed & 85 & 15 & 8 & 2 & 36 & 18 \\
\hline & $\ldots$ & Dimethenamid + metribuzin & 87 & 15 & 11 & 1 & 36 & 18 \\
\hline & $\ldots$ & Pendimethalin & 86 & 15 & 11 & 1 & 36 & 18 \\
\hline & $\ldots$ & Acifluorfen & 88 & 15 & 11 & 2 & 36 & 18 \\
\hline & $\ldots$ & Imazethapyr & 87 & 15 & 11 & 2 & 36 & 18 \\
\hline & $\ldots$ & $\mathrm{LSD}_{0.05}$ & NS & NS & NS & NS & NS & NS \\
\hline
\end{tabular}

a Percent seed germination.

b 100 -seed weight.

c Incidence of seed infected with Phomopsis spp.

${ }^{\mathrm{d}}$ Incidence of seed infected with Cercospora kikuchii.

e Protein concentration.

${ }^{\mathrm{f}}$ Fisher's protected least significant difference at $\alpha=0.05$.

g Not significant at $P \leq 0.05$.

Table 3. Quality parameters of soybean seed harvested at DeKalb, IL

\begin{tabular}{|c|c|c|c|c|c|c|}
\hline Year & Cultivar & Herbicide & Germination $(\%)^{\mathbf{a}}$ & Seed weight $(g)^{b}$ & Phomopsis spp. $(\%)^{\mathrm{c}}$ & Cercospora kikuchii $(\%)^{\mathrm{d}}$ \\
\hline \multirow[t]{11}{*}{1997} & BSR 101 & $\ldots$ & 80 & 24 & 5 & 2 \\
\hline & Jack & $\ldots$ & 94 & 22 & 3 & 3 \\
\hline & Pioneer 9254 & $\ldots$ & 98 & 21 & 1 & 0 \\
\hline & Savoy & $\ldots$ & 96 & 20 & 1 & 1 \\
\hline & $\operatorname{LSD}_{0.05} \mathrm{e}^{\mathrm{e}}$ & $\ldots$ & 4 & $\mathrm{NS}^{\mathrm{f}}$ & 2 & NS \\
\hline & $\ldots$ & Hand weed & 91 & 22 & 1 & 2 \\
\hline & $\ldots$ & Pendimethalin & 90 & 23 & 3 & 1 \\
\hline & $\ldots$ & Acifluorfen & 94 & 21 & 3 & 2 \\
\hline & $\ldots$ & Acifluorfen + bentazon & 93 & 20 & 3 & 2 \\
\hline & $\ldots$ & Imazethapyr & 91 & 22 & 3 & 1 \\
\hline & $\cdots$ & $\mathrm{LSD}_{0.05}$ & NS & NS & NS & NS \\
\hline \multirow[t]{11}{*}{1998} & BSR 101 & $\ldots$ & 86 & 17 & 40 & 0 \\
\hline & Jack & $\ldots$ & 94 & 14 & 22 & 1 \\
\hline & Pioneer 9254 & $\ldots$ & 96 & 14 & 20 & 0 \\
\hline & Savoy & $\ldots$ & 94 & 18 & 11 & 0 \\
\hline & $\operatorname{LSD}_{0.05}$ & $\ldots$ & 2 & 1 & 6 & NS \\
\hline & $\ldots$ & Hand weed & 91 & 16 & 25 & 0 \\
\hline & $\ldots$ & Pendimethalin & 92 & 16 & 21 & 0 \\
\hline & $\ldots$ & Acifluorfen & 94 & 16 & 24 & 0 \\
\hline & $\ldots$ & Acifluorfen + bentazon & 93 & 16 & 23 & 1 \\
\hline & $\ldots$ & Imazethapyr & 92 & 16 & 22 & 0 \\
\hline & $\ldots$ & $\mathrm{LSD}_{0.05}$ & NS & NS & NS & NS \\
\hline
\end{tabular}

a Percent seed germination.

b 100 -seed weight.

${ }^{\mathrm{c}}$ Incidence of seed infected with Phomopsis spp.

${ }^{\mathrm{d}}$ Incidence of seed infected with Cercospora kikuchii.

e Fisher's protected least significant difference at $\alpha=0.05$.

${ }^{\text {f }}$ Not significant at $P \leq 0.05$. 
kikuchii incidence and protein and oil concentration. There were no significant differences among cultivars for 100-seed weight. There was significant variation among herbicides for 100-seed weight. Plots sprayed with acifluorfen had the greatest 100 -seed weight $(16 \mathrm{~g})$. Herbicides did not have a significant effect on seed germination, incidence of Phomopsis spp. or C. kikuchii, or protein and oil concentration.

Urbana. In 1998, there was significant $(P \leq 0.05)$ variation among cultivars for seed germination, 100-seed weight, incidence of Phomopsis spp., and protein and oil concentration (Table 2). Asgrow 3704 and Pioneer 9363 had the significantly greatest seed germination (94\%). Savoy had the significantly greatest 100-seed weight (18 g). Incidence of Phomopsis spp. was greatest in Savoy (15\%), but did not significantly differ from Jack (12\%). Protein concentration was greatest in Asgrow 3904, Pioneer 9363, and Savoy (36\%), and oil concentration was greatest in Asgrow 3704 and Jack (20\%). Incidence of C. $\mathrm{ki}$ kuchii did not significantly differ among cultivars. Herbicides did not have a significant effect on the seed quality parameters measured.

In 1999, there was significant $(P \leq 0.05)$ variation among cultivars for seed germination, 100-seed weight, incidence of Phomopsis spp. and C. kikuchii, and protein and oil concentration (Table 2). Asgrow 3704 had the significantly greatest seed germination (93\%). Savoy had the significantly greatest 100 -seed weight (17 g). Jack and Savoy had the greatest incidence of Phomopsis spp. (17 and 16\%,
Table 5. Pearson correlation coefficients between seed quality parameters of soybean seed harvested at Champaign, $\mathrm{IL}^{\mathrm{a}}$

\begin{tabular}{|c|c|c|c|c|}
\hline \multirow{2}{*}{$\frac{\text { Year }}{1998}$} & \multicolumn{2}{|c|}{ Variables } & \multirow{2}{*}{$\begin{array}{c}\begin{array}{c}\text { Pearson correlation } \\
\text { coefficient }\end{array} \\
-0.28\end{array}$} & \multirow{2}{*}{$\frac{\text { Probability }}{0.0056}$} \\
\hline & Germination $^{\mathrm{b}}$ & Phomopsis spp. ${ }^{\mathrm{c}}$ & & \\
\hline & Germination & Cercospora kikuchii ${ }^{\mathrm{d}}$ & -0.04 & 0.6885 \\
\hline & Germination & Seed weight ${ }^{\mathrm{e}}$ & -0.02 & 0.8551 \\
\hline & Germination & Protein & 0.40 & 0.0001 \\
\hline & Germination & Oil & -0.28 & 0.0067 \\
\hline & Phomopsis spp. & C. kikuchii & -0.02 & 0.8583 \\
\hline & Phomopsis spp. & Seed weight & 0.34 & 0.0007 \\
\hline & Phomopsis spp. & Protein & -0.17 & 0.0954 \\
\hline & Phomopsis spp. & Oil & 0.18 & 0.0807 \\
\hline & C. kikuchii & Seed weight & -0.01 & 0.9253 \\
\hline & C. kikuchii & Protein & -0.25 & 0.0164 \\
\hline & C. kikuchii & Oil & 0.21 & 0.0463 \\
\hline & Seed weight & Protein & 0.10 & 0.3296 \\
\hline & Seed weight & Oil & -0.02 & 0.8799 \\
\hline & Protein & Oil & -0.79 & 0.0001 \\
\hline \multirow[t]{15}{*}{1999} & Germination & Phomopsis spp. & -0.42 & 0.0001 \\
\hline & Germination & C. kikuchii & -0.06 & 0.5643 \\
\hline & Germination & Seed weight & 0.03 & 0.7941 \\
\hline & Germination & Protein & -0.02 & 0.8465 \\
\hline & Germination & Oil & 0.04 & 0.6888 \\
\hline & Phomopsis spp. & C. kikuchii & 0.00 & 0.9727 \\
\hline & Phomopsis spp. & Seed weight & 0.07 & 0.4916 \\
\hline & Phomopsis spp. & Protein & 0.20 & 0.0447 \\
\hline & Phomopsis spp. & Oil & 0.25 & 0.0111 \\
\hline & C. kikuchii & Seed weight & -0.03 & 0.7861 \\
\hline & C. kikuchii & Protein & 0.15 & 0.1265 \\
\hline & C. kikuchii & Oil & -0.27 & 0.0073 \\
\hline & Seed weight & Protein & 0.20 & 0.0443 \\
\hline & Seed weight & Oil & 0.03 & 0.7615 \\
\hline & Protein & Oil & -0.07 & 0.4747 \\
\hline
\end{tabular}

${ }^{a}$ Correlation coefficients were based on 100 observations each year.

${ }^{b}$ Percent seed germination.

c Incidence of seed infected with Phomopsis spp.

${ }^{\mathrm{d}}$ Incidence of seed infected with Cercospora kikuchii.

e 100-seed weight.

Table 4. Quality parameters of soybean seed harvested at Monmouth, IL

\begin{tabular}{|c|c|c|c|c|c|c|}
\hline Year & Cultivar & Herbicide & Germination $(\%)^{\mathbf{a}}$ & Seed weight $(g)^{b}$ & Phomopsis spp. $(\%)^{\mathrm{c}}$ & Cercospora kikuchii $(\%)^{\mathrm{d}}$ \\
\hline \multirow[t]{10}{*}{1999} & Asgrow 3002 & $\ldots$ & 91 & 15 & 4 & 1 \\
\hline & Pioneer 93B01 & $\ldots$ & 96 & 13 & 0 & 0 \\
\hline & Pioneer 9363 & $\ldots$ & 93 & 14 & 11 & 1 \\
\hline & Siebens 2701 & $\ldots$ & 89 & 13 & 1 & 3 \\
\hline & $\mathrm{LSD}_{0.05}{ }^{\mathrm{e}}$ & $\ldots$ & 3 & 1 & 5 & 2 \\
\hline & $\ldots$ & Glyphosate only ${ }^{\mathrm{f}}$ & 92 & 14 & 2 & 0 \\
\hline & $\ldots$ & Pendimethalin & 93 & 14 & 6 & 1 \\
\hline & $\ldots$ & Acifluorfen & 93 & 14 & 4 & 1 \\
\hline & $\ldots$ & Imazethapyr & 92 & 14 & 3 & 2 \\
\hline & $\ldots$ & $\mathrm{LSD}_{0.05}$ & $\mathrm{NS}^{\mathrm{g}}$ & NS & NS & NS \\
\hline \multirow[t]{10}{*}{2000} & Asgrow 3002 & $\ldots$ & 85 & 14 & 0 & 2 \\
\hline & Pioneer 93B01 & $\ldots$ & 92 & 12 & 3 & 2 \\
\hline & Pioneer 9363 & $\ldots$ & 90 & 12 & 1 & 3 \\
\hline & Siebens 2701 & $\ldots$ & 88 & 13 & 1 & 3 \\
\hline & $\mathrm{LSD}_{0.05}$ & $\ldots$ & 3 & 1 & 2 & NS \\
\hline & $\ldots$ & Glyphosate only & 89 & 12 & 0 & 3 \\
\hline & $\ldots$ & Pendimethalin & 89 & 12 & 2 & 3 \\
\hline & $\ldots$ & Acifluorfen & 89 & 12 & 2 & 3 \\
\hline & $\ldots$ & Imazethapyr & 88 & 13 & 1 & 3 \\
\hline & $\ldots$ & $\mathrm{LSD}_{0.05}$ & NS & NS & NS & NS \\
\hline
\end{tabular}

\footnotetext{
a Percent seed germination.

b 100 -seed weight.

c Incidence of seed infected with Phomopsis spp.

${ }^{\mathrm{d}}$ Incidence of seed infected with Cercospora kikuchii.

e Fisher's protected least significant difference at $\alpha=0.05$.

${ }^{f}$ Glyphosate was applied on the entire study to control weeds.

${ }^{\mathrm{g}}$ Not significant at $P \leq 0.05$.
} 
respectively). Jack had the greatest incidence of C. kikuchii (4\%). Savoy had the greatest protein concentration $(37 \%)$. Asgrow 3704, Pioneer 9363, and Savoy had the greatest oil concentration $(19 \%)$. Herbicides did not have a significant effect on the seed quality parameters measured.

DeKalb. In 1997, there was significant $(P \leq 0.05)$ variation among cultivars for seed germination and incidence of Phomopsis spp. (Table 3). Pioneer 9254 had significantly greater seed germination (98\%) than all of the other cultivars except for Savoy (96\%). Incidence of Phomopsis spp. was greatest in BSR 101 (5\%). There were no significant differences among cultivars for 100 -seed weight and incidence of $C$. kikuchii. Herbicides did not have a significant effect on the seed quality parameters measured.

In 1998 , there was significant $(P \leq 0.05)$ variation among cultivars for seed germination, 100-seed weight, and incidence of Phomopsis spp. (Table 3). Pioneer 9254 had the significantly greatest seed germination (96\%). Savoy had the significantly greatest 100-seed weight (18 g). BSR 101 had the significantly greatest incidence of Phomopsis spp. (40\%). There were no significant differences among cultivars for incidence of $C$. kikuchii. Herbicides did not have a significant effect on the seed quality parameters measured.

Monmouth. In 1999, there was significant $(P \leq 0.05)$ variation among cultivars for seed germination, 100-seed weight, and incidence of Phomopsis spp. and C. kikuchii (Table 4). Pioneer 93B01 had significantly greater germination $(96 \%)$ than the other cultivars. Asgrow 3002 had significantly greater 100-seed weight $(15 \mathrm{~g})$ than the other cultivars. Pioneer 9363 had the significantly highest incidence of Phomopsis spp. (11\%). Siebens 2701 had the greatest incidence of C. kikuchii (3\%). Herbicides did not have a significant effect on the seed quality parameters measured.

In 2000, there was significant $(P \leq 0.05)$ variation among cultivars for seed germination, 100-seed weight, and incidence of Phomopsis spp. (Table 4). Pioneer 93B01 had significantly greater germination $(92 \%)$ than the other cultivars except for Pioneer 9363 (90\%). Asgrow 3002 had the greatest 100 -seed weight $(14 \mathrm{~g})$. Pioneer 93B01 had the greatest incidence of Phomopsis spp. (3\%). There were no significant differences among cultivars for incidence of $C$. kikuchii. Herbicides did not

Table 6. Pearson correlation coefficients between seed quality parameters of soybean seed harvested at Urbana, $\mathrm{IL}^{\mathrm{a}}$

\begin{tabular}{|c|c|c|c|c|}
\hline \multirow{2}{*}{$\frac{\text { Year }}{1998}$} & \multicolumn{2}{|c|}{ Variables } & \multirow{2}{*}{$\begin{array}{c}\text { Pearson correlation } \\
\text { coefficient }\end{array}$} & \multirow{2}{*}{$\begin{array}{c}\text { Probability } \\
0.0604\end{array}$} \\
\hline & Germination $^{\mathrm{b}}$ & Phomopsis spp. ${ }^{\mathrm{c}}$ & & \\
\hline & Germination & Cercospora kikuchii ${ }^{\mathrm{d}}$ & $\mathrm{ND}^{\mathrm{f}}$ & ND \\
\hline & Germination & Seed weight ${ }^{\mathrm{e}}$ & -0.01 & 0.9102 \\
\hline & Germination & Protein & 0.28 & 0.0046 \\
\hline & Germination & Oil & -0.04 & 0.7039 \\
\hline & Phomopsis spp. & C. kikuchii & ND & ND \\
\hline & Phomopsis spp. & Seed weight & 0.36 & 0.0002 \\
\hline & Phomopsis spp. & Protein & 0.02 & 0.8115 \\
\hline & Phomopsis spp. & Oil & -0.08 & 0.4575 \\
\hline & C. kikuchii & Seed weight & ND & ND \\
\hline & C. kikuchii & Protein & ND & ND \\
\hline & C. kikuchii & Oil & ND & ND \\
\hline & Seed weight & Protein & 0.41 & 0.0001 \\
\hline & Seed weight & Oil & -0.36 & 0.0003 \\
\hline & Protein & Oil & -0.76 & 0.0001 \\
\hline \multirow[t]{15}{*}{1999} & Germination & Phomopsis spp. & -0.38 & 0.0001 \\
\hline & Germination & C. kikuchii & -0.21 & 0.0380 \\
\hline & Germination & Seed weight & -0.03 & 0.7733 \\
\hline & Germination & Protein & -0.18 & 0.0813 \\
\hline & Germination & Oil & -0.05 & 0.6001 \\
\hline & Phomopsis spp. & C. kikuchii & -0.02 & 0.8506 \\
\hline & Phomopsis spp. & Seed weight & 0.01 & 0.9550 \\
\hline & Phomopsis spp. & Protein & 0.12 & 0.2307 \\
\hline & Phomopsis spp. & Oil & 0.05 & 0.6449 \\
\hline & C. kikuchii & Seed weight & -0.23 & 0.0188 \\
\hline & C. kikuchii & Protein & -0.12 & 0.2368 \\
\hline & C. kikuchii & Oil & 0.02 & 0.8397 \\
\hline & Seed weight & Protein & 0.35 & 0.0003 \\
\hline & Seed weight & Oil & -0.07 & 0.4909 \\
\hline & Protein & Oil & -0.26 & 0.0091 \\
\hline
\end{tabular}

a Correlation coefficients were based on 100 observations each year.

b Percent seed germination.

${ }^{c}$ Incidence of seed infected with Phomopsis spp.

${ }^{\mathrm{d}}$ Incidence of seed infected with Cercospora kikuchii.

e 100 -seed weight.

${ }^{\mathrm{f}}$ Not determinable due to no detectable incidence of $C$. kikuchii from seed harvested at Urbana, IL in 1998. have a significant effect on the seed quality parameters measured.

Correlations. At Champaign in 1998, there were significant $(P \leq 0.05)$ negative correlations between seed germination and incidence of Phomopsis spp., between seed germination and oil concentration, between incidence of $C$. kikuchii and protein concentration, and between protein concentration and oil concentration (Table 5). There were significant positive correlations between seed germination and protein concentration, between incidence of Phomopsis spp., and between incidence of $C$. kikuchii and oil concentration.

At Champaign in 1999, there were significant $(P \leq 0.05)$ negative correlations between seed germination and incidence of Phomopsis spp. and between incidence of C. kikuchii and oil concentration (Table 5). There were significant positive correlations between incidence of Phomopsis spp. and protein concentration, between incidence of Phomopsis spp. and oil concentration, and between 100-seed weight and protein concentration.

At Urbana in 1998, there were significant $(P \leq 0.05)$ negative correlations between 100-seed weight and protein concentration, and between protein concentration and oil concentration (Table 6). There were significant positive correlations between seed germination and protein concentration, between incidence of Phomopsis spp. and 100-seed weight, and between 100seed weight and protein concentration.

At Urbana in 1999, there were significant $(P \leq 0.05)$ negative correlations between seed germination and incidence of Phomopsis spp., between seed germination and incidence of $C$. kikuchii, between incidence of $C$. kikuchii and 100-seed weight, and between protein concentration and oil concentration (Table 6). There was a significant positive correlation between 100 -seed weight and protein concentration.

At DeKalb in 1997, there was a significant $(P \leq 0.05)$ negative correlation between seed germination and incidence of Phomopsis spp. (Table 7). At DeKalb in 1998, there were significant negative correlations between seed germination and incidence of Phomopsis spp., and between seed germination and 100-seed weight.

At Monmouth in 1999, there were no significant $(P \leq 0.05)$ correlations (Table $8)$. At Monmouth in 2000 , there was a significant negative correlation between seed germination and 100-seed weight.

\section{DISCUSSION}

In our studies, herbicides did not increase or decrease the concentration of protein or oil within soybean seeds. This agrees with other reports that herbicides did not affect protein and/or oil concentration in soybean seeds $(18,28,38)$. These previous studies, reported from 1969 to 1973, tested some herbicides that are no longer used in soybean production in the 
United States. In our studies, herbicides from relatively newer herbicide classes were included (i.e. diphenyl ethers, imidazolinones). From this and other research, it appears that the use of herbicides does not alter important soybean seed components, indicating that soybean growers should choose herbicides based on economic reasons and the population of weed species present in a field, and not be concerned with the effects on seed composition. The management of weeds in soybean fields is still an important factor in obtaining good seed quality, in that it reduces the population of other hosts of soybean seed pathogens $(15,23,25)$, and reduces competition that might affect seed size and overall yield. The study presented herein was designed to test direct effects of herbicides on soybean seed quality. The indirect effect of herbicides controlling weeds, which may serve as hosts or contribute to a more conducive microenvironment for seed pathogens to infect was not tested in these studies, but has been documented $(5,8,10,12)$.
Tested under four field environments, Asgrow 3704 ranked first for seed germination each time. Savoy, which was tested under six field environments, ranked either first or second for highest incidence of Phomopsis spp. at Champaign and Urbana for both years (15 to 24\%), but had the least incidence of Phomopsis spp. at DeKalb for both years (1 to $11 \%$ ). BSR 101 had the greatest incidence of Phomopsis spp. both years at Dekalb (5 and 40\%). When Savoy, which is MG II, was planted at Champaign and Urbana, most of the other cultivars were MG III. At DeKalb, all cultivars were MG II, except for BSR 101, which is MG I. This agrees with other reports that earlier maturing cultivars may develop more Phomopsis infected seed if environmental conditions are conducive $(21,40,42,45)$.

There were significant $(P \leq 0.05)$ inverse relationships between incidence of seed infected with Phomopsis spp. and seed germination in seed from Champaign, Urbana, and DeKalb, which is a typical reaction of soybean seed infected with

Table 7. Pearson correlation coefficients between seed quality parameters of soybean seed harvested at DeKalb, $\mathrm{IL}^{\mathrm{a}}$

\begin{tabular}{|c|c|c|c|c|}
\hline \multirow{2}{*}{$\frac{\text { Year }}{1997}$} & \multicolumn{2}{|c|}{ Variables } & \multirow{2}{*}{$\begin{array}{c}\begin{array}{c}\text { Pearson correlation } \\
\text { coefficient }\end{array} \\
-0.53\end{array}$} & \multirow{2}{*}{$\frac{\text { Probability }}{0.0001}$} \\
\hline & Germination $^{\mathrm{b}}$ & Phomopsis spp. ${ }^{\mathrm{c}}$ & & \\
\hline & Germination & Cercospora kikuchii & -0.00 & 0.9903 \\
\hline & Germination & Seed weight ${ }^{\mathrm{e}}$ & -0.20 & 0.0828 \\
\hline & Phomopsis spp. & C. kikuchii & 0.13 & 0.2372 \\
\hline & Phomopsis spp. & Seed weight & 0.05 & 0.6648 \\
\hline & C. kikuchii & Seed weight & -0.07 & 0.5399 \\
\hline \multirow[t]{6}{*}{1998} & Germination & Phomopsis spp. & -0.56 & 0.0001 \\
\hline & Germination & C. kikuchii & 0.07 & 0.5160 \\
\hline & Germination & Seed weight & -0.28 & 0.0109 \\
\hline & Phomopsis spp. & C. kikuchii & -0.06 & 0.5856 \\
\hline & Phomopsis spp. & Seed weight & -0.06 & 0.5967 \\
\hline & C. kikuchii & Seed weight & -0.10 & 0.3883 \\
\hline
\end{tabular}

\footnotetext{
a Correlation coefficients were based on 80 observations each year.

${ }^{\mathrm{b}}$ Percent seed germination.

${ }^{c}$ Incidence of seed infected with Phomopsis spp.

${ }^{\mathrm{d}}$ Incidence of seed infected with Cercospora kikuchii.

e 100 -seed weight.
}

Table 8. Pearson correlation coefficients between seed quality parameters of soybean seed harvested at Monmouth, $\mathrm{IL}^{\mathrm{a}}$

\begin{tabular}{|c|c|c|c|c|}
\hline \multirow{2}{*}{$\frac{\text { Year }}{1999}$} & \multicolumn{2}{|c|}{ Variables } & \multirow{2}{*}{$\begin{array}{c}\begin{array}{c}\text { Pearson correlation } \\
\text { coefficient }\end{array} \\
-0.04\end{array}$} & \multirow{2}{*}{$\begin{array}{c}\text { Probability } \\
0.7728\end{array}$} \\
\hline & Germination $^{\mathrm{b}}$ & Phomopsis spp. ${ }^{\mathrm{c}}$ & & \\
\hline & Germination & Cercospora kikuchii ${ }^{\mathrm{d}}$ & -0.20 & 0.1230 \\
\hline & Germination & Seed weight ${ }^{\mathrm{e}}$ & -0.10 & 0.4356 \\
\hline & Phomopsis spp. & C. kikuchii & 0.06 & 0.6542 \\
\hline & Phomopsis spp. & Seed weight & 0.19 & 0.1329 \\
\hline & C. kikuchii & Seed weight & -0.06 & 0.6617 \\
\hline \multirow[t]{6}{*}{2000} & Germination & Phomopsis spp. & 0.07 & 0.5855 \\
\hline & Germination & C. kikuchii & -0.07 & 0.5698 \\
\hline & Germination & Seed weight & -0.60 & 0.0001 \\
\hline & Phomopsis spp. & C. a kikuchii & 0.15 & 0.2168 \\
\hline & Phomopsis spp. & Seed weight & -0.15 & 0.2347 \\
\hline & C. kikuchii & Seed weight & 0.05 & 0.6728 \\
\hline
\end{tabular}

\footnotetext{
${ }^{\text {a }}$ Correlation coefficients were based on 64 observations each year.

${ }^{\mathrm{b}}$ Percent seed germination.

${ }^{c}$ Incidence of seed infected with Phomopsis spp.

${ }^{\mathrm{d}}$ Incidence of seed infected with Cercospora kikuchii.

${ }^{\mathrm{e}} 100$-seed weight.
}

Phomopsis spp. (22). There was a significant positive relationship detected between incidence of Phomopsis spp. and soybean seed weight at Champaign and Urbana in 1998. Hepperly and Sinclair (16) and Mbuvi et al. (25), however, reported that soybean seeds showing symptoms of $P$. longicolla infection weighed less than asymptomatic seeds. In our studies, a significant relationship between incidence of seed infected with Phomopsis spp. and concentration of protein or oil within soybean seeds was detected at Champaign in 1999 only. This relationship was positive, which agrees with Hepperly and Sinclair (16) who reported that seeds with symptoms of Phomopsis spp. infection contained more oil and protein than asymptomatic seeds; however, Velicheti et al. (43) found that $P$. longicolla was able to degrade seed proteins. The severity of infection, which is influenced by environment $(1,19,20,29,30,33,37,41)$, could play a role in determining how seed weight and constituents such as protein and oil are affected. Additionally, not all infected seeds show symptoms, therefore, this could account for different interpretations between studies that measure infection vs. symptoms.

The incidence of seed with $C$. kikuchii was very low at each location, which limits the conclusions that can be drawn from correlations between $C$. kikuchii infection and seed quality parameters. There was a significant $(P \leq 0.05)$ positive correlation between $C$. kikuchii and oil concentration at Champaign in 1998, but there was also a significant negative relationship between the same two variables at Champaign in 1999. C. kikuchii has been reported to reduce seed germination $(27,46)$ and oil concentration $(27,39)$, but the relationship is unclear from our study due to a lack of disease. BSR 101, which has been reported as being susceptible to C. kikuchii (26), was planted at the DeKalb location, and although it produced seed with poor germination, it did not differ from the other cultivars in seed infection by C. kikuchii.

From our research, it appears that Phomopsis spp. may be a more prevalent seed pathogen than $C$. kikuchii for soybean fields in central to northern Illinois. We feel that both pathogens are important, however, and that with severe infection they may be able to inflict considerable damage to seeds. Damages caused by these pathogens are not only important to soybean growers, but are also important to seed companies and food processors.

\section{ACKNOWLEDGMENTS}

The authors thank the Soybean Research and Development Council and the Illinois Soybean Program Operating Board for partial funding of this project; W. Batchelor and N. Boedhram for overseeing protein and oil analysis of seed; and E. Adee, K. Ames, D. Maxwell, D. Mueller, and L. Paul for technical assistance. 


\section{LITERATURE CITED}

1. Balducchi, A. J. and McGee, D. C. 1987. Environmental factors influencing infection of soybean seeds by Phomopsis and Diaporthe species during seed maturation. Plant Dis. 71:209-212

2. Barnett, H. L. and Hunter, B. B. 1998. Illustrated Genera of Imperfect Fungi, 4th ed. American Phytopathological Society, St. Paul, $\mathrm{MN}$

3. Bauske, E. M. and Kirby, H. W. 1992. Effect of dinitroaniline herbicides, carboxin-pentachloronitrobenzene seed treatment, and Rhizoctonia disease on soybean. Plant Dis. 76:236-239.

4. Black, B. D., Russin, J. S., Griffin, J. L., and Snow, J. P. 1996. Herbicide effects on Rhizoctonia solani in vitro and Rhizoctonia foliar blight of soybean (Glycine max). Weed Sci. 44:711-716.

5. Bowman, J. E., Hartman, G. L., McClary, R. D., Sinclair, J. B., Hummel, J. W., and Wax, L. M. 1986. Effects of weed control and row spacing in conventional tillage, reduced tillage, and nontillage on soybean seed quality. Plant Dis. 70:673-676.

6. Bowman, J. E. and Sinclair, J. B. 1989. Effect of herbicides on Rhizoctonia seedling disease of soybeans in glasshouse experiments. J. Phytopathol. 124:267-274.

7. Bradley, C. A., Hartman, G. L., Wax, L. M., and Pedersen, W. L. Influence of herbicides on Rhizoctonia root and hypocotyl rot of soybean. Crop Prot. (In press).

8. Bradley, C. A., Wax, L. M., Ebelhar, S. A., Bollero, G. A., and Pedersen, W. L. 2001. The effect of fungicide seed protectants, seeding rates, and reduced rates of herbicides on notill soybean. Crop Prot. 20:615-622.

9. Canaday, C. H., Helsel, D. G., and Wyllie, T. D. 1986. Effects of herbicide-induced stress on root colonization of soybeans by Macrophomina phaseolina. Plant Dis. 70:863-866.

10. Chagas, D., and Dhingra, O. D. 1979. Effect of timing of weed control on the incidence of seedborne fungi in dry bean seeds. Fitopatol. Bras. 4:423-426.

11. Dann, E. K., Diers, B. W., and Hammerschmidt, R. 1999. Suppression of Sclerotinia stem rot of soybean by lactofen herbicide treatment. Phytopathology 89:598-602.

12. Dhingra, O. D., and da Silva, J. F. 1978. Effect of weed control on the internally seedborne fungi in soybean seeds. Plant Dis. Rep. 62:513-516.

13. Fehr, W. R, Caviness, C. E., Burmood, D. T., and Pennington, J. S. 1971. Stage of development descriptions for soybeans, Glycine $\max ($ L.) Merrill. Crop Sci. 11:929-931.

14. Hardcastle, W. S., Wilkinson, R. F., and Young, C. T. 1974. Metribuzin effects on seed constituents of soybean varieties. Weed Sci. 22:575-577.

15. Hepperly, P. R., Kirkpatrick, B. L., and Sinclair, J. B. 1980. Abutilon theophrasti: wild host for three fungal parasites of soybean.
Plant Dis. 70:307-310.

16. Hepperly, P. R., and Sinclair, J. B. 1978. Quality losses in Phomopsis-infected soybean seeds. Phytopathology 68:1684-1687.

17. Hobbs, T. W., Schmitthenner, A. F., and Kuter, G. A. 1985. A new Phomopsis species from soybean. Mycologia 77:534-544.

18. Johnson, B. J. and Jellum, M. D. 1969. Effect of pesticides on chemical composition of soybean seed [Glycine max (L.) Merrill]. Agron. J. 61:379-380.

19. Jordan, E. G., Manandhar, J. B., Thapliyal, P. N., and Sinclair, J. B. 1986. Factors affecting soybean seed quality in Illinois. Plant Dis. 70:246-248.

20. Jordan, E. G., Sinclair, J. B., Manandhar, J. B., and Thapliyal, P. N. 1992. Soil type and other field conditions affecting seed-borne fungi in Illinois soyabeans. Seed Sci. Technol. 20:619-628.

21. Kmetz, K. T., Schmitthenner, A. F., and Ellett, C. W. 1978. Soybean seed decay: prevalence of infection and symptom expression caused by Phomopsis sp., Diaporthe phaseolorum var. sojae, and D. phaseolorum var. caulivora. Phytopathology 68:836-840.

22. Kulik, M. M., and Sinclair, J. B. 1999. Phomopsis seed decay. Pages 31-32 in: Compendium of Soybean Diseases, 4th ed. G. L. Hartman, J. B. Sinclair, and J. C. Rupe, eds. American Phytopathological Society, St. Paul, $\mathrm{MN}$.

23. Li, S., Bradley, C. A., Hartman, G. L., and Pedersen, W. L. 2001. First report of Phomopsis longicolla from velvetleaf causing stem lesions on inoculated soybean and velvetleaf plants. Plant Dis. 85:1031.

24. Mbuvi, S. W., Litchfield, J. B., and Sinclair, J. B. 1989. Physical properties of soybean seeds damaged by fungi and a virus. Trans. Am. Soc. Agric. Eng. 32:2093-2096

25. McLean, K. S and Roy, K. W. 1988. Purple seed stain of soybean caused by isolates of Cercospora kikuchii from weeds. Can. J. Plant Pathol. 10:166-171.

26. Orth, C. E., and Schuh, W. 1994. Resistance of 17 soybean cultivars to foliar, latent, and seed infection by Cercospora kikuchii. Plant Dis. 78:661-664.

27. Pathan, M. A., Sinclair, J. B., and McClary, R. D. 1989. Effects of Cercospora kikuchii on soybean seed germination and quality. Plant Dis. 73:720-723.

28. Penner, D., and Meggitt, W. F. 1970. Herbicide effects on soybean [Glycine $\max$ (L.) Merrill] seed lipids. Crop Sci. 10:553-555.

29. Rupe, J. C. 1990. Effect of temperature on the rate of infection of soybean seedlings by Phomopsis longicolla. Can. J. Plant Pathol. 12:43-47.

30. Rupe, J. C. and Ferriss, R. S. 1986. Effects of pod moisture on soybean seed infection by Phomopsis sp. Phytopathology 76:273-277.

31. Sanogo, S., Yang, X. B., and Lundeen, P. 2001. Field response of glyphosate-tolerant soybean to herbicides and sudden death syndrome. Plant Dis. 85:773-779.

32. Sanogo, S., Yang, X. B., and Scherm, H. 2000. Effects of herbicides on Fusarium solani f. sp. glycines and development of sudden death syndrome in glyphosate-tolerant soybean. Phytopathology 90:57-66.

33. Shortt, B. J., Grybauskas, A. P., Tenne, F. D., and Sinclair, J. B. 1981. Epidemiology of Phomopsis seed decay of soybean in Illinois. Plant Dis. 65:62-64.

34. Sinclair, J. B. 1992. Discoloration of soybean seeds-an indicator of quality. Plant Dis. 76:1087-1091.

35. Sinclair, J. B. 1993. Phomopsis seed decay of soybeans-a prototype for studying seed disease. Plant Dis. 77:329-334.

36. Sinclair, J. B. 1995. Reevaluation of grading standards and discounts for fungus-damaged soybean seeds. J. Am. Oil Chem. Soc. 72:1415-1419.

37. Spilker, D. A., Schmitthenner, A. F., and Ellett, C. W. 1981. Effects of humidity, temperature, fertility, and cultivar on the reduction of soybean seed quality by Phomopsis sp Phytopathology 71:1027-1029.

38. Stoller, E. W., Weber, E. J., and Wax, L. M. 1973. The effects of herbicides on soybean seed constituents. J. Environ. Qual. 2:241-244.

39. Taira, H., Taira, H., Kokubu, Y., Otake, S. and Takezaki, C. 1980. Chemical composition on purple specked soybean seeds by Cercospora kikuchii. Rep. Nat. Food Res. Inst. 37:16-24.

40. Tekrony, D. M., Egli, D. B., Balles, J., Tomes, L., and Stuckey, R. E. 1984. Effect of date of harvest maturity on soybean seed quality and Phomopsis sp. seed infection. Crop Sci. 24:189-193.

41. TeKrony, D. M., Egli, D. B., Stuckey, R. E., and Balles, J. 1983. Relationship between weather and soybean seed infection by Phomopsis sp. Phytopathology 73:914-918.

42. Thomison, P. R., Kenworthy, W. J., and McIntosh, M. S. 1990. Phomopsis seed decay in soybean isolines differing in stem termination, time of flowering, and maturity. Crop Sci. 30:183-188.

43. Velicheti, R. K., Kollipara, K. P., Sinclair, J. B., and Hymowitz, T. 1992. Selective degradation of proteins by Cercospora kikuchii and Phomopsis longicolla in soybean seed coats and cotyledons. Plant Dis. 76:779-782.

44. Wilcox, J. R., and Abney, T. S. 1973. Effects of Cercospora kikuchii on soybeans. Phytopathology 63:796-797.

45. Wrather, J. A., Kendig, S. R., Wiebold, W. J., and Riggs, R. D. 1996. Cultivar and planting date effects on soybean stand, yield, and $P h o$ mopsis sp. seed infection. Plant Dis. 80:622 624.

46. Yeh, C. C., and Sinclair, J. B. 1982. Effect of Cercospora kikuchii on soybean seed germination and its interaction with Phomopsis sp. Phytopathol. Z. 105:265-270. 